\title{
Non-degenerate Umbilics, the Path Formulation and Gradient Bifurcation Problems
}

\author{
Jacques-Elie Furter * \\ Dep. of Mathematical Sciences \\ Brunel University \\ Uxbridge UB8 3PH, UK
}

\author{
Angela Maria Sitta ${ }^{\dagger}$ \\ UNESP - Universidade Estadual Paulista, \\ Departamento de Matemática - IBILCE, \\ Campus de São José do Rio Preto - SP, Brazil
}

March 21, 2004

\begin{abstract}
Parametrised contact-equivalence is successful for the understanding and classification of the qualitative local behaviour of bifurcation diagrams and their perturbations. Path formulation is an alternative point of view. It makes explicit the singular behaviour due to the core of the bifurcation germ (when the parameters vanish) from the effects of the way parameters enter.

Here we show how path formulation can be used to classify and structure efficiently multiparameter bifurcation problems in corank 2 problems. In particular, the non degenerate umbilics singularities are the generic cores in four situations: the general or gradient problems and the $\mathbb{Z}_{2}$-equivariant (general or gradient) problems where $\mathbb{Z}_{2}$ acts on the second component of $\mathbb{R}^{2}$ via $\kappa(x, y)=(x,-y)$. The universal unfolding of the umbilic singularities have an interesting 'Russian doll' type of structure of universal unfoldings in all those categories.

In our approach we can handle one, or more, parameter situations using the same framework. We can even consider some special parameter structure (for instance some internal hierarchy). We classify the generic bifurcations with 1, 2 or 3 parameters that occur in those cases. Some results are known with one bifurcation parameter, but the others are new.

We discuss some application to the bifurcation of a cylindrical panel under different loads structure. This problem has many natural parameters that provide concrete examples of our generic diagrams around the first interaction of the buckling modes.
\end{abstract}

\section{Introduction}

In this work we discuss multiparameter bifurcation problems $f(x, \lambda)=0$ of corank two based around a non degenerate quadratic part $f(x, 0)$ in the state variables. The theory of parametrised contact-equivalence of Golubitsky-Schaeffer [14] has been very successful for the understanding and classification of the qualitative local behaviour of bifurcation diagrams and their perturbations. By bifurcation diagrams we mean the zero-set of parametrised equations of the type $f(x, \lambda)=0$ where $x$ represent the state space and $\lambda$ the bifurcation parameter(s). Both are finite dimensional or we assume a reduction of Lyapunov-Schmidt type applies. For the local behaviour we mean that

*partially supported by FAPESP

${ }^{\dagger}$ FAPESP, processo 03/03107-09 
we consider germs near the origin. Two bifurcation germs $f, g$ are $\mathcal{K}_{\lambda}$-equivalent if there exist (orientation preserving) changes of coordinates $(T, X, L)$ around the origin such that

$$
g(x, \lambda)=T(x, \lambda) f(X(x, \lambda), L(\lambda))
$$

Clearly $(T, X, L)$ induces a local diffeomorphism between the zero-sets of $f$ and $g$ and preserves the special role of the bifurcation parameters (cf. [14, 15]). In $[20,2,9,10]$ an alternative point of view as been developed: the path formulation. In this work we aim to show how path formulation can be used to classify and structure efficiently multiparameter bifurcation problems. It organises $\mathcal{K}_{\lambda}$-equivalence by allowing to distinguish the singular behaviour due to the core of the bifurcation germ from the effects of the parameters. The core of a bifurcation problem $f(x, \lambda)=0$ is the germ $f_{0}(x)=f(x, 0)$ obtained by setting the parameters equal to zero. It represents the singular behaviour independently of the way the parameter(s) enter. In corank 1 many results appear in our references (cf. [9] for a comprehensive account). Here we are interested in corank 2 problems. In particular, non degenerate umbilics singularities are the generic cores in four situations: the general or gradient problems with or without a $\mathbb{Z}_{2}$-symmetry where $\mathbb{Z}_{2}$ acts on the second component of $\mathbb{R}^{2}$ via $\kappa(x, y)=(x,-y)$.

The non degenerate umbilic singularities $f_{0}:\left(\mathbb{R}^{2}, 0\right) \rightarrow \mathbb{R}^{2}$ are defined by

$$
f_{0}(x, y)=\left(\begin{array}{c}
\epsilon_{1} x^{2}+\epsilon_{2} y^{2} \\
2 \epsilon_{3} x y
\end{array}\right)
$$

where $\epsilon_{i}^{2}=1, i=1,2,3$. We discuss aspects of the path formulation for bifurcation problems based on $f_{0}$. More precisely, to a bifurcation problem $f:\left(\mathbb{R}^{2} \times \mathbb{R}^{k}, 0\right) \rightarrow \mathbb{R}^{2}$ with $k$ bifurcation parameters and core $f_{0}$, we associate a path $\bar{\alpha}:\left(\mathbb{R}^{k}, 0\right) \rightarrow \mathbb{R}^{a}$ in the parameter space $\mathbb{R}^{a}$ of the universal unfolding $F_{a}$ of $f_{0}$ in the relevant category $\left(\mathcal{K}\right.$, gradient or $\left.\mathcal{K}^{\mathbb{Z}_{2}}\right)$ such that $f$ and the pullback $\bar{\alpha}^{*} F_{a}$ are $\mathcal{K}_{\lambda}$-equivalent (cf. Section 1.1 for details). Then the description of such bifurcation problems and their deformations can be broadly understood as deformations of paths via changes of coordinates respecting the discriminant of the projection of $F_{a}^{-1}(0)$ onto the parameter space $\mathbb{R}^{a}$. Further details will be explained later in the introduction.

In $[12,11]$ we have developed the technical points needed to investigate such problems. In this work we classify the generic bifurcations with 1, 2 or 3 parameters that occur in those cases using the path formulation for bifurcation problems, study some gradient bifurcation diagrams and their perturbations and consider a buckling problem as an example. Some classification results are known. The generic corank two case has been studied in [14] and some of the gradient cases in [24]. Later, in [2], a comprehensive theory was developed but the examples were concentrated on the equivariant gradient case. There is an extensive classification of $\mathbb{Z}_{2}$-equivariant bifurcation germs in [7]. One advantage of our approach is that we can handle one, two, or more, parameter situations using the same framework. We can even consider some special parameter structure (for instance some internal hierarchy, cf. Theorem 6 and $[9,10,11])$. In Section 3 we classify

1. the generic one parameter bifurcation germs of corank two in our four categories (recovering and extending previously known results),

2. as new results, the generic two parameter bifurcation germs of corank two,

3. and discuss a case with 3 parameters and a hierarchy of parameters. 
The stability of solutions, given by the eigenvalues structure of the linearisation along branches, is an important information in bifurcation theory. To take care of all possible cases we should consider umbilics with negative coefficients for $x^{2}$ but, by using the change of coordinates $f \rightarrow-f$, we exchange the stability assignments on the branches and so we can work with (2.5) only without limiting the generality.

As examples we use a modification of a model of [23] of the bifurcation of a cylindrical panel under different loads. This problem has many natural parameters that provide concrete examples of our generic diagrams around the first interaction of the buckling modes. In Section 4 we look at two specific cases to illustrate our classifications:

1. a one bifurcation parameter gradient problem,

2. a $\mathbb{Z}_{2}$-equivariant two parameter problem.

We finish this introduction with more details on the principles of path equivalence and their applications to the classification of gradient bifurcation problems.

\subsection{Principles of Path Equivalence}

Given a bifurcation germ $f$ we construct the path $\bar{\alpha}$ representing it by considering $f$ as an unfolding with parameters $\lambda$ of the core $f_{0}$ in the relevant category. Let $F_{a}$ be the universal unfolding of $f_{0}$ in such category. The theory of unfolding then means that

$$
f(x, \lambda)=T(x, \lambda) F_{a}(X(x, \lambda), \bar{\alpha}(\lambda))
$$

for some $\bar{\alpha}$. This means that $f$ and the pull back $\bar{\alpha}^{*} F_{a}$ are $\mathcal{K}_{\lambda}$-equivalent with equivalence $(T, X, I)$. The qualitative study of the zero set of bifurcation problems with the same core is obtained through the study of their associated paths, in particular their position with respect to the discriminant variety $\Delta^{F_{a}}$ associated with $F_{a}$. More precisely, let $\pi_{F_{a}}:\left(F_{a}^{-1}(0), 0\right) \rightarrow \mathbb{R}^{a}$ be the restriction of the natural projection $\pi:\left(\mathbb{R}^{n+a}, 0\right) \rightarrow \mathbb{R}^{a}$. Then $\Delta^{F_{a}}=\pi_{F_{a}}\left(\Sigma_{F_{a}}\right)$ where $\Sigma_{F_{a}}$ is the local bifurcation set of $F_{a}$. Clearly $\Delta^{F_{a}}$ monitors when, and 'how', a path $\bar{\alpha}$ induces a crossing of $\Sigma_{F_{a}}$, that is, when there is a local change in behaviour of the zero-set. In Section 2 we define those varieties in more detail. We prefer to choose $\Delta^{F_{a}}$ as the real slice of the discriminant of the complexification of $F_{a}$. This means that we can complexify the situation and use the power of singularity theory in the complex realm. For finite codimension problems we do not loose anything.

The idea of the path formulation goes back at least to Arnold [1] and was the original starting point of the work [13]. In there the very fruitful $\mathcal{K}_{\lambda}$-equivalence approach had finally been developed because the technicalities of the path formulation could not be easily overcome at the time. The ideas behind the path formulation were resurrected in $[19,20]$ for the usual contact-equivalence and in [2] for (symmetric) gradient problems. It followed recent progresses in singularity theory allowing to handle variety preserving contact-equivalence. Since then, an algebraic formulation has been derived in [8] which shows that the main features of the path formulation occur naturally in the algebra of $\mathcal{K}_{\lambda}$-theory via the concept of liftable vector fields (cf. [4]). Fix a universal unfolding of $F_{a}$ of $f_{0}$ in the appropriate category. We say that the two paths $\bar{\alpha}, \bar{\beta}:\left(\mathbb{R}^{k}, 0\right) \rightarrow\left(\mathbb{R}^{a}, 0\right)$ are path equivalent if

$$
\bar{\alpha}(\lambda)=H(\lambda, \bar{\beta}(L(\lambda)))
$$

where $L:\left(\mathbb{R}^{k}, 0\right) \rightarrow\left(\mathbb{R}^{k}, 0\right)$ is an orientation preserving diffeomorphism and $H:\left(\mathbb{R}^{k+a}, 0\right) \rightarrow\left(\mathbb{R}^{a}, 0\right)$ is a $\lambda$-parametrised family of local diffeomorphism on $\mathbb{R}^{a}$ path connected to the identity that 
preserves the discriminant $\Delta^{F_{a}}$ of $F_{a}$ in the sense that $H\left(\lambda, \Delta^{F_{a}}\right) \subset \Delta^{F_{a}}$ for $\lambda \in\left(\mathbb{R}^{k}, 0\right)$. More precisely, we choose $\Delta^{F_{a}}$ as the real slice of the discriminant of the complexification of $F_{a}$ (cf. [10]). For a fixed $F_{a}$, the set of path equivalences $\mathcal{K}_{\Delta}^{F_{a}}$ form a geometric subgroup of $\mathcal{K}$ in the sense of Damon's, hence the usual theory and calculations of singularity apply. Note that we cannot in general simplify $H$ in (1.2) as a $\lambda$-parametrised matrix as with the usual $\mathcal{K}$-equivalence and an explicit description of the diffeomorphisms $H$ is in general impossible. Nevertheless, the tangent spaces of paths can be determined explicitly at lower order or with the help of computer algebra packages. In particular, the extended tangent space of a path $\bar{\alpha}$ is given by the $\mathcal{E}_{\lambda}$-module

$$
<\bar{\alpha}_{\lambda}>_{\mathcal{E}_{\lambda}}+\bar{\alpha}^{*}\left(\operatorname{Derlog}\left(\Delta^{F_{a}}\right)\right)_{\mathcal{E}_{\lambda}}
$$

where $\operatorname{Derlog}\left(\Delta^{F_{a}}\right)$ is the $\mathcal{E}_{\alpha}$-module of vector fields tangent to the discriminant $\Delta^{F_{a}}$ (cf. Section 2 ). We denote by $\mathcal{E}_{z}$ the ring of smooth germs $f:\left(\mathbb{R}^{n}, 0\right) \rightarrow \mathbb{R}$ with variable $z$, by $\overrightarrow{\mathcal{E}}_{z}$ the $\mathcal{E}_{z}$-module of smooth germs $f:\left(\mathbb{R}^{n}, 0\right) \rightarrow \mathbb{R}^{m}$ when $m$ is clear from the context. We denote by $\mathcal{O}_{z}$, $\overrightarrow{\mathcal{O}}_{z}$, the same rings, modules, of analytic germs. Let $R$ be a local ring, we denote by $\left\langle m_{1} \ldots m_{k}>_{R}\right.$ the $R$-module generated by the $m_{i}$ 's. Note that (1.2) is the definition of contact equivalence of sections over $\Delta^{F_{a}}$. In general we need to use the subgroup of diffeomorphisms liftable over $F_{a}^{-1}(0)$ (cf. $[8,10])$. In our contexts, both groups indeed coincide.

\subsection{Variational Bifurcation}

In [2] we have derived a theory for (equivariant) gradient bifurcation problems. Let $g:\left(\mathbb{R}^{n} \times \mathbb{R}^{k}, 0\right) \rightarrow$ $\mathbb{R}$ be a germ, we say that $\nabla_{x} g(x, \lambda)=0$ is a gradient bifurcation problem when $\nabla_{x} g(0,0)=0$ and $\nabla_{x}^{2} g(0,0)=0$. Its potential is $g$. Gradient bifurcation problems form an $\mathcal{E}_{\lambda}$-submodule of $\overrightarrow{\mathcal{E}}_{(x, \lambda)}$ we denote by $\overrightarrow{\mathcal{E}}_{\nabla}=\left\{\nabla_{x} g(x, \lambda) \mid g \in \mathcal{E}_{(x, \lambda)}\right\}$. Contact equivalence is also an equivalence relation on $\overrightarrow{\mathcal{E}}_{\nabla}$. But, for an arbitrary contact equivalence, $(T, X, L) \cdot \nabla_{x} f$ is not necessarily in $\overrightarrow{\mathcal{E}}_{\nabla}$. Therefore some modification of the usual techniques is necessary in order to describe the contact classes and their perturbations inside $\overrightarrow{\mathcal{E}}_{\nabla}$.

A natural framework for such problems is right equivalence for potentials with some special consideration for the parameter $\lambda: f, g \in \mathcal{E}_{(x, \lambda)}$ are equivalent if there exists a change of coordinates $(X, L)$ such that

$$
f(x, \lambda)=g(X(x, \lambda), L(\lambda)) .
$$

Although this theory has an elegant simplicity, it turns out to be inadequate. Clearly, if $f, g$ satisfy (1.4) then $\nabla_{x} f$ is contact equivalent to $\nabla_{x} g$ but the converse is not true in general. There are two distinct difficulties involving different levels of complexity.

The first obstruction is linked with the difference between contact equivalence $(\mathcal{K})$ of gradients and right equivalence $(\mathcal{R})$ of potentials and does not involve the distinguished parameter. In particular $\mathcal{R}$-equivalence can introduce moduli (parameters that cannot be scaled away by a smooth change of coordinates) that are irrelevant in the context of $\mathcal{K}$-equivalence. Examples are the $T_{p, q, r}$ singularities, $x^{p}+y^{q}+z^{r}+m x y z$ with integer exponent such that $\frac{1}{p}+\frac{1}{q}+\frac{1}{r}<1$. The parameter $m$ is an $\mathcal{R}$-moduli but can be scaled away in a $\mathcal{K}$-equivalence of the gradients.

A second difficulty is more fundamental: the change of coordinates $(X, L)$ in (1.4) is too restrictive with the distinguished parameter $\lambda$. Singularities of infinite codimension arise immediately. For example, the potential $f_{1}(x, \lambda)=\frac{1}{4} x^{4}+\frac{1}{2} \lambda x^{2}$ of the pitchfork is of infinite $\mathcal{R}_{\lambda}$-codimension but $\nabla_{x} f_{1}$ is of $\mathcal{K}_{\lambda}$-codimension 2. A similar, more immediate, fact is that $x^{4}+x^{3}+\lambda x$ is $\mathcal{K}_{\lambda}$-contact equivalent to $x^{3}+\lambda x$ but, at the potential level, $\frac{1}{5} x^{5}+\frac{1}{4} x^{4}+\frac{1}{2} \lambda x^{2}$ is not $\mathcal{R}_{\lambda}$-equivalent to $\frac{1}{4} x^{4}+\frac{1}{2} \lambda x^{2}$. 
The first obstruction is more a nuisance than a difficulty: it introduces unnecessary distinctions in the classification. The second is fundamental as it precludes finite codimension. In some cases we can use left-right equivalence $\left(\mathcal{A}_{\lambda}\right)$ with parameters, but this is again not satisfactory. The $\mathcal{A}_{\lambda}$-codimension increases much more rapidly than the $\mathcal{K}_{\lambda}$-codimension, and, in most cases the $\mathcal{A}_{\lambda}$-codimension is still infinite.

Another approach suggested in [13] is to use the concept of paths in the parameter space. It has been extended and applied to some one parameter bifurcation problems in $\mathbb{R}^{2}$ by Zuppa [24]. In those two works the path formulation is used with the $\mathcal{R}$-universal unfolding of the core $f_{0}$. But this still does not solve the problem of the appearance of unwanted moduli in the classification of the cores. For these reasons we took a hybrid approach in [2]. We developed a theory based on the 'gradient' part of the tangent spaces used in the classical approach. Although there is not an a priori group of change of coordinates, the principal results go through. In the present context the two theories coincide and the path formulation goes through applied to the $\mathcal{R}$-unfolding of the umbilics when $\gamma=0$.

\section{Cores and Derlogs}

A $\mathcal{K}$-miniversal unfolding of $f_{0}$ in the general corank two category is $F_{0}:\left(\mathbb{R}^{2} \times \mathbb{R}^{4}, 0\right) \rightarrow \mathbb{R}^{2}$ (of codimension 4) defined as

$$
F_{0}\left(x, y, \alpha_{1}, \alpha_{2}, \beta, \gamma\right)=\left(\begin{array}{c}
x^{2}+\epsilon y^{2}+\alpha_{1} x+\alpha_{2}+\gamma y \\
2 \epsilon x y-\alpha_{1} \epsilon y+\beta-\gamma x
\end{array}\right)
$$

The universal unfoldings of $f_{0}$ in the other categories are imbedded into $F_{0}$. When $\gamma=0,(2.5)$ is the universal unfolding in the gradient case (cf. Section 1.2) and when $\gamma=\beta=0$ in the $\mathbb{Z}_{2^{-}}$ equivariant cases (both $\mathcal{K}^{\mathbb{Z}_{2}}$ and gradient). Note that when $\epsilon=-1$ and $\alpha_{2}=\beta=\gamma=0$, (2.5) is also the universal unfolding for the generic $\mathbb{D}_{3}$-equivariant core $\left(\mathbb{D}_{3}\right.$ acts as the group of isometry of the equilateral triangle). The universal unfolding of the umbilic singularities have therefore an interesting 'Russian doll' type of structure of universal unfoldings in all those categories ([12]).

We denote by $\nabla_{x} G_{0}$ the restriction of $F_{0}$ to the gradient situation, $\gamma=0$, of potential

$$
G_{0}\left(x, y, \alpha_{1}, \alpha_{2}, \beta\right)=\frac{1}{3} x^{3}+\epsilon x y^{2}+\frac{1}{2} \alpha_{1}\left(x^{2}-\epsilon y^{2}\right)+\alpha_{2} x+\beta y .
$$

It represents the $\mathcal{R}$-universal unfolding of $f_{0}$ of codimension 3. Because $G_{0}$ is quasi-homogeneous, $\nabla_{x} G_{0}$ is also the gradient universal unfolding of $\nabla_{x} f_{0}$ (cf. [2]). With the $\mathbb{Z}_{2}$-symmetry, $F_{0}^{\mathbb{Z}_{2}}$ is the restriction of $F_{0}$ when $\beta=\gamma=0$ :

$$
F_{0}^{\mathbb{Z}_{2}}\left(x, y, \alpha_{1}, \alpha_{2}\right)=\left(\begin{array}{c}
x^{2}+\epsilon y^{2}+\alpha_{1} x+\alpha_{2} \\
2 \epsilon x y-\epsilon \alpha_{1} y
\end{array}\right) .
$$

Note that $F_{0}^{\mathbb{Z}_{2}}$ is also the gradient of the $\mathbb{Z}_{2}$-invariant potential

$$
\frac{1}{3} x^{3}+\epsilon x y^{2}+\frac{1}{2} \alpha_{1}\left(x^{2}-\epsilon y^{2}\right)+\alpha_{2} x .
$$

Therefore, in both $\mathbb{Z}_{2}$-equivariant cases, the problem is of codimension 2 . This equality means that the gradient and dissipative theories for the non degenerate umbilics are equal with a $\mathbb{Z}_{2}$-symmetry. We recall what is needed is the next section. 


\section{$2.1 \mathbb{Z}_{2}$-Equivariant Problems}

The ring of $\mathbb{Z}_{2}$-invariant germs is generated by $x$ and $v=y^{2}$ and the module of equivariant germs is freely generated over the ring of invariant germs by $(1,0)$ and $(0, y)$. A $\mathbb{Z}_{2}$-equivariant map $F$ on $\mathbb{R}^{2}$, with parameter $a$, has components $P(x, v, a)$ and $y Q(x, v, a)$. It is a gradient if and only if $2 P_{v} \equiv Q_{x}$ and if $G$ is a $\mathbb{Z}_{2}$-invariant potential then $F(z, a)=\nabla_{z} G(z, a)=\left(G_{x}, 2 y G_{v}\right)$, so $P=G_{x}$ and $Q=2 G_{v}$. The solution set consists of two pieces, distinguished by the symmetry of the solution:

- $\operatorname{Fix}\left(\mathbb{Z}_{2}\right)$ of equation $P(x, 0, a)=0$. The eigenvalues of $F_{z}(x, 0, a)$ are $P_{x}(x, 0, a)$ and $Q(x, 0, a)$. The local bifurcation varieties are $\mathcal{B}_{x}=\left\{(x, 0, a) \mid P(x, 0, a)=P_{x}(x, 0, a)=0\right\}$ and $\mathcal{P}_{\kappa}=$ $\{(x, 0, a) \mid P(x, 0, a)=Q(x, 0, a)=0\}$.

- $\operatorname{Fix}(\mathbf{1})$ of equation $P(x, v, a)=Q(x, v, a)=0$. The eigenvalues of $F_{z}(z, a)$ satisfy $\operatorname{tr} F_{z}=$ $P_{x}+2 v Q_{v}$ and $\operatorname{det} F_{z}=2 v\left(P_{x} Q_{v}-Q_{x} P_{v}\right)$. The local bifurcation variety $\mathcal{B}_{\kappa}$ satisfies the equations $P(x, v, a)=Q(x, v, a)=P_{x}(x, v, a) Q_{v}(x, v, a)-Q_{x}(x, v, a) P_{v}(x, v, a)=0$.

There are possible Hopf bifurcation points near the roots of $P(x, v, a)=Q(x, v, a)=P_{x}(x, v, a)+$ $2 v Q_{v}(x, v, a)=0$ satisfying $P_{x} Q_{v}-Q_{x} P_{v}>0$. But those points are not invariant of the contact equivalence. When $f$ is not a gradient, we can ascertain their existence using continuity arguments along branches of solutions where the determinant does not change sign but the trace does (cf. $[14])$.

Proposition 1 The non-degenerate umbilics are the generic cores in the $\mathbb{Z}_{2}$-equivariant general and gradient cases with the same universal unfolding.

Proof. For all cases the calculation is classic ([15]). We use the usual techniques for (equivariant)

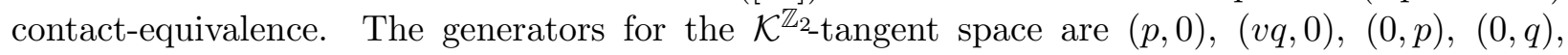
$\left(v p_{v}, v q_{v}\right)$ and $\left(p_{x}, q_{x}\right)$. The first and last one generated on $\mathcal{M}_{(x, v)}$ to eliminate the higher order terms.

\subsection{Discriminants}

The local bifurcation set of $F_{0}$ is

$$
\Sigma_{F_{0}}=\left\{(x, y, \alpha, \beta, \gamma) \mid F_{0}(x, y, \alpha, \beta, \gamma)=0 \text { and } d_{(x, y)} F_{0}(x, y, \alpha, \beta, \gamma) \text { is singular }\right\} .
$$

It is a manifold of dimension 4 constituted generically of fold points. The discriminant $\Delta^{F_{0}}$ of $F_{0}$ is the variety of reduced equation $h=0$ where $h(\alpha, \beta, \gamma)$ is equal to

$$
\begin{aligned}
& \left(\alpha_{1}^{2}-4 \alpha_{2}\right)\left(3 \alpha_{1}^{2}+4 \alpha_{2}\right)^{3}+32 \epsilon \beta^{2}\left(9 \alpha_{1}^{4}-48 \alpha_{1}^{2} \alpha_{2}+16 \alpha_{2}^{2}\right)-256 \beta^{4} \\
& -1024\left(\beta^{2}+3 \epsilon \alpha_{2}^{2}\right) \alpha_{1} \beta \gamma-4\left(144 \alpha_{1}^{2} \beta^{2}+384 \alpha_{2} \beta^{2}+27 \epsilon \alpha_{1}^{6}-144 \epsilon \alpha_{1}^{2} \alpha_{2}^{2}+128 \epsilon \alpha_{2}^{3}\right) \gamma^{2} \\
& +18\left(9 \alpha_{1}^{4}-16 \alpha_{2}^{2}+16 \epsilon \beta^{2}\right) \gamma^{4}-108 \epsilon \alpha_{1}^{2} \gamma^{6}+27 \gamma^{8} .
\end{aligned}
$$

The local bifurcation set of $G_{0}$ is $\Sigma_{G_{0}}=\left\{(x, y, \alpha, \beta) \mid \nabla_{x} G_{0}(x, y, \alpha, \beta)\right.$ is singular $\}$. It is a manifold of dimension 3 also constituted generically of fold points. The discriminant $\Delta^{G_{0}}$ of $G_{0}$ is the variety of equation

$$
h^{\nabla}(\alpha, \beta)=\left(\alpha_{1}^{2}-4 \alpha_{2}\right)\left(3 \alpha_{1}^{2}+4 \alpha_{2}\right)^{3}+32 \epsilon \beta^{2}\left(9 \alpha_{1}^{4}-48 \alpha_{1}^{2} \alpha_{2}+16 \alpha_{2}^{2}\right)-256 \beta^{4}=0 .
$$


The discriminant $\Delta^{\mathbb{Z}_{2}}$ of $F_{0}^{\mathbb{Z}_{2}}$ is formed from the projections of the local bifurcation varieties $\mathcal{P}_{\kappa}$ and $\mathcal{B}_{x}$, generically formed of pitchforks or folds, respectively. Together the equation of the (reducible but principal) discriminant is

$$
h^{\mathbb{Z}_{2}}(\alpha)=\left(3 \alpha_{1}^{2}+4 \alpha_{2}\right)\left(\alpha_{1}^{2}-4 \alpha_{2}\right)=0 .
$$

\subsection{Derlogs and Liftable Vector Fields}

For the equivalence of the singularity theories for finite codimension bifurcation germs and their associated paths we actually need the notion of vector fields liftable via the projections $\pi_{F_{a}}$. Without limiting the generality we can consider analytic germs when dealing with finite codimension problems. Therefore we can complexify the problem and use the whole power of algebraic geometry $([6,4])$.

A vector field germ $\xi:\left(\mathbb{C}^{a}, 0\right) \rightarrow \mathbb{C}^{a}$ is liftable over $\pi_{F_{a}}$ if there exists a vector field germ $\eta:\left(\mathbb{C}^{2+a}, 0\right) \rightarrow \mathbb{C}^{2}$ and a matrix map germ $T:\left(\mathbb{C}^{2+a}, 0\right) \rightarrow \mathrm{M}(2, \mathbb{C})$ in the right category such that

$$
\left(F_{a}\right)_{z}(z, \alpha) \eta(z, \alpha)+\left(F_{a}\right)_{\alpha}(z, \alpha) \xi(\alpha)=T(z, \alpha) F_{a}(z, \alpha) .
$$

This definition is geometric in the sense that $\xi$ lifts to vector fields $(\eta, \xi)$ tangent to $F_{a}^{-1}(0)$ at its smooth points. In our problems the liftable vector fields are exactly the vector fields tangent to the discriminant. Let $\mathcal{I}\left(\Delta^{F_{a}}\right)$ denote the ideal of germs vanishing on $\Delta^{F_{a}}$. Define $\operatorname{Derlog}\left(\Delta^{F_{a}}\right)=\{\xi \in$ $\left.\overrightarrow{\mathcal{O}}_{a} \mid \xi\left(\mathcal{I}\left(\Delta^{F_{a}}\right)\right) \subset \mathcal{I}\left(\Delta^{F_{a}}\right)\right\}$. It extends to the coherent sheaf of vector fields tangent to $\Delta^{F_{a}}$ because it can also be defined as the kernel of an epimorphism of coherent modules (cf. [10] and references). The discriminant $\Delta^{F_{a}}$ is a free (or Saito) divisor if $\operatorname{Der} \log \left(\Delta^{F_{a}}\right)$ is a locally free $\mathcal{O}_{a}$-module (of rank $a)$. To calculate the generators of $\operatorname{Derlog}\left(\Delta^{F_{a}}\right)$ we use the following result.

Theorem 2 (Saito [22]) (a) If the vector fields $\left\{\xi_{i}\right\}_{i=1}^{a}$ are in $\operatorname{Derlog}\left(\Delta^{F_{a}}\right)$ and the determinant $\left|\xi_{1} \ldots \xi_{a}\right|$ is a reduced defining equation for $\Delta^{F_{a}}$ then those vector fields generate freely $\operatorname{Derlog}\left(\Delta^{F_{a}}\right)$.

(b) If the vector fields $\left\{\xi_{i}\right\}_{i=1}^{a}$ form a Lie algebra and $\left|\xi_{1} \ldots \xi_{a}\right|=0$ is a reduced defining equation for a hypersurface $\Delta$ of $\mathbb{C}^{a}$ then they generate freely $\operatorname{Derlog}(\Delta)$.

We therefore obtain the following result.

Theorem 3 ([12]) (a) The Derlog $\Delta^{F_{0}}$ is freely generated over $\mathcal{O}_{(\alpha, \beta, \gamma)}$ by the nilpotent basis

$$
\begin{aligned}
& \xi_{1}=\left(\alpha_{1}, 2 \alpha_{2}, 2 \beta, \gamma\right), \\
& \xi_{2}=\left(3 \epsilon \gamma,-2 \epsilon \beta,-2 \alpha_{2}, 3 \alpha_{1}\right), \\
& \xi_{3}=\left(8 \alpha_{2},-8 \alpha_{1} \alpha_{2}+3 \alpha_{1}^{3}-3 \epsilon \alpha_{1} \gamma^{2}-8 \epsilon \beta \gamma, 8 \alpha_{2} \gamma+8 \alpha_{1} \beta+3 \epsilon \gamma^{3}-3 \alpha_{1}^{2} \gamma,-8 \beta\right), \\
& \xi_{4}=\left(-8 \epsilon \beta, 3 \gamma^{3}-3 \epsilon \alpha_{1}^{2} \gamma-8 \epsilon \alpha_{1} \beta-8 \epsilon \alpha_{2} \gamma, 8 \alpha_{1} \alpha_{2}+8 \epsilon \beta \gamma+3 \alpha_{1}^{3}-3 \epsilon \alpha_{1} \gamma^{2}, 8 \alpha_{2}\right) .
\end{aligned}
$$

A nilpotent basis consists of an Euler field (like $\xi_{1}$ ) and a basis of the annihilator of $h\left(\Delta^{F_{0}}\right.$ has equation $h=0)$. Every element in the Derlog lifts.

(b) The Derlog of $\Delta^{G_{0}}$ is generated by the following

$$
\begin{aligned}
& \phi_{1}=\left(\alpha_{1}, 2 \alpha_{2}, 2 \beta\right), \\
& \phi_{2}=\left(8 \alpha_{2}^{2}-8 \epsilon \beta^{2},-8 \alpha_{1} \alpha_{2}^{2}+3 \alpha_{1}^{3} \alpha_{2}-8 \epsilon \alpha_{1} \beta^{2}, 16 \alpha_{1} \alpha_{2} \beta+3 \alpha_{1}^{3} \beta\right), \\
& \phi_{3}=\left(24 \alpha_{1} \alpha_{2},-16 \epsilon \beta^{2}-24 \alpha_{1}^{2} \alpha_{2}+9 \alpha_{1}^{4},-16 \alpha_{2} \beta+24 \alpha_{1}^{2} \beta\right), \\
& \phi_{4}=\left(24 \epsilon \alpha_{1} \beta,-16 \epsilon \alpha_{2} \beta+24 \epsilon \alpha_{1}^{2} \beta,-\left(4 \alpha_{2}+3 \alpha_{1}^{2}\right)^{2}\right) .
\end{aligned}
$$


Note that $\operatorname{Derlog}\left(\Delta^{G_{0}}\right)$ is not freely generated over $\mathcal{O}_{(\alpha, \beta)}$ but it is a Lie sub-algebra of $\operatorname{Der} \log \left(\Delta^{F_{0}}\right)$. Moreover, $\phi_{1}$ is an Euler field and $\phi_{2}, \phi_{3}, \phi_{4}$ are in the annihilator of $h^{\nabla}$ and every element in the Derlog lifts.

(c) The Derlog of $\Delta^{\mathbb{Z}_{2}}$ is freely generated over $\mathcal{O}_{\alpha}$ by the nilpotent basis

$$
\zeta_{1}=\left(\alpha_{1}, 2 \alpha_{2}\right), \quad \zeta_{2}=\left(8 \alpha_{2}+2 \alpha_{1}^{2}, 3 \alpha_{1}^{3}-4 \alpha_{1} \alpha_{2}\right)
$$

and every element in the Derlog lifts.

\section{Generic Bifurcation Problems}

\subsection{One Bifurcation Parameter}

Without the gradient conditions, corank two problems with one bifurcation parameter are of high codimension; the simplest one is codimension 3 (the so-called "hill-top" bifurcation, [14]), then we jump to topological codimension 5 and higher (cf. [13, 14]). Some results for gradient bifurcation problems are available from Zuppa [24]. The coefficients $\delta, \delta_{1}$ are \pm 1 . In (3.12) $m$ is a modal parameter and satisfy $m^{2} \neq \epsilon$. Recall that a modal parameter is an invariant of smooth contact equivalence. The parameters $\hat{\beta}_{i}$ 's are the unfolding parameters.

Theorem 4 (a) The generic bifurcation problem of corank two with one bifurcation parameter have normal form

$$
f(x, y, \lambda)=\left(x^{2}+\epsilon y^{2}+\delta \lambda, 2 \epsilon x y\right),
$$

when $\epsilon=-1$. When $\epsilon=1$, there is another additional normal form

$$
f(x, y, \lambda)=\left(x^{2}+y^{2}, 2 x y+\delta \lambda\right) .
$$

Normal form (3.8) occurs when $\left|\left(f_{1}\right)_{\lambda}^{o}\right|^{2}>\left|\left(f_{2}\right)_{\lambda}^{o}\right|^{2}$, as normal form (3.9) occurs when $\left|\left(f_{1}\right)_{\lambda}^{o}\right|^{2}<$ $\left|\left(f_{2}\right)_{\lambda}^{o}\right|^{2}$. The condition is higher when the two coefficients are equal.

Both are of codimension 3 with universal unfolding

$$
F(x, y, \lambda, \hat{\beta})=\left(\begin{array}{c}
x^{2}+\epsilon y^{2}+\hat{\beta}_{1} x+\hat{\beta}_{3} y+\delta \lambda \\
2 \epsilon x y-\epsilon \hat{\beta}_{1} y-\hat{\beta}_{3} x+\hat{\beta}_{2}
\end{array}\right),
$$

for (3.8) and

$$
F(x, y, \lambda, \hat{\beta})=\left(\begin{array}{c}
x^{2}+\epsilon y^{2}+\hat{\beta}_{1} x+\hat{\beta}_{3} y+\hat{\beta}_{2} \\
2 \epsilon x y-\epsilon \hat{\beta}_{1} y-\hat{\beta}_{3} x+\delta \lambda
\end{array}\right)
$$

for (3.9).

(b) The generic gradient bifurcation problem of corank two with one bifurcation parameter has normal form

$$
f(x, y, \lambda)=\left(x^{2}+\epsilon y^{2}+\delta_{1} \lambda x+\delta \lambda, 2 \epsilon x y-\epsilon \delta_{1} \lambda y+m \lambda\right) .
$$

It is of topological codimension 2 with universal unfolding

$$
F(x, y, \lambda, \hat{\beta})=\left(\begin{array}{c}
x^{2}+\epsilon y^{2}+\left(\delta_{1} \lambda+\hat{\beta}_{1}\right) x+\delta \lambda \\
2 \epsilon x y-\left(\epsilon \delta_{1} \lambda+\hat{\beta}_{1}\right) y+m \lambda+\hat{\beta}_{2}
\end{array}\right) .
$$




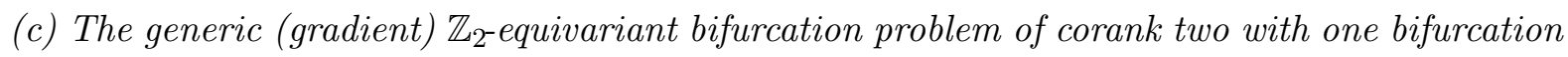
parameter has normal form

$$
f(x, y, \lambda)=\left(x^{2}+\epsilon y^{2}+\delta \lambda, 2 \epsilon x y\right)
$$

It is of codimension 1 with universal unfolding

$$
F(x, y, \lambda, \hat{\beta})=\left(\begin{array}{c}
x^{2}+\epsilon y^{2}+\hat{\beta} x+\delta \lambda \\
2 \epsilon x y-\epsilon \hat{\beta} y
\end{array}\right) \text {. }
$$

Proof. (a) We are going to prove that the generic path in the general corank two case is $(0, \delta \lambda, 0,0)$ of universal unfolding $\left(\hat{\beta}_{1}, \delta \lambda, \hat{\beta}_{2}, \hat{\beta}_{3}\right)$. First note that the path $\bar{\alpha}(\lambda)=(a \lambda, b \lambda, c \lambda, d \lambda)+\overrightarrow{\mathcal{M}}_{\lambda}^{2}$ is of codimension 3 and the quadratic terms in $\lambda$ are in its unipotent tangent space if $c^{2}-\epsilon b^{2} \neq 0$. We can use Nakayama's Lemma on the terms of lower order of the generators of $\operatorname{Derlog}\left(\Delta^{F_{a}}\right)$ :

$$
\begin{aligned}
\bar{\alpha}_{\lambda} & =(a, b, c, d) \\
\xi_{1} & =(a \lambda, 2 b \lambda, 2 c \lambda, d \lambda) \\
\xi_{2} & =(3 \epsilon d \lambda,-2 \epsilon c \lambda,-2 b \lambda, 3 a \lambda) \\
\xi_{3} & =(b \lambda, 0,0,-c \lambda) \\
\xi_{4} & =(-\epsilon c \lambda, 0,0, b \lambda)
\end{aligned}
$$

modulo $\mathcal{M}_{\lambda}^{2}$. The condition is always satisfied for either $b$ or $c$ non zero if $\epsilon=-1$ and for $b \neq \pm c$ if $\epsilon=1$. This corresponds to the conditions (H1) and (H3) of [14] (p. 403). In those cases we can change coordinates to set-up $b=\delta= \pm 1$ and $a=c=d=0$.

(b) We proceed like in (a). Let $\bar{\alpha}(\lambda)=(a \lambda, b \lambda, c \lambda)+\overrightarrow{\mathcal{M}}_{\lambda}^{2}$. The tangent space is generated by $(a, b, c),(0, b \lambda, c \lambda),\left(3 \epsilon a c \lambda^{2},-2 \epsilon b c \lambda^{2},-2 b^{2} \lambda^{2}\right),\left(3 a b \lambda^{2},-2 \epsilon c^{2} \lambda^{2},-2 b c \lambda^{2}\right)$ and $\left(\left(b^{2}-\epsilon c^{2}\right) \lambda^{2}, 0,0\right)$. With the same conditions as in (a), namely $c^{2}-\epsilon b^{2} \neq 0$, the quadratic terms in $\lambda^{2}$ are contained in the unipotent tangent space of $\bar{\alpha}$. Moreover, the topological codimension of $\bar{\alpha}$ is larger or equal to 3 if $a=0$, so in the generic case $a \neq 0$. Via re-scaling we find the final result as $c$ cannot be now eliminated and so becomes a modal parameter.

(c) We proceed in a similar way with $\bar{\alpha}(\lambda)=(a \lambda, b \lambda)+\overrightarrow{\mathcal{M}}_{\lambda}^{2}$ and the generators $(a, b),(0, b \lambda)$ and $(b \lambda, 0)$ modulo $\mathcal{M}_{\lambda}^{2}$. When $b \neq 0$ the quadratic terms can be removed from the unipotent tangent space as well as $a$.

\subsection{Two and Three Bifurcation Parameters}

In [21] one can find a classification of corank 1 two parameter bifurcation germs. To our knowledge, no results in corank two are previously available. We denote the bifurcation parameters by $\Lambda=$ $(\lambda, \mu)$. As previously, the coefficients $\delta_{i}$ are \pm 1 . In (b) $m$ is modal and $m\left(m^{2}-\epsilon\right) \neq 0$. The parameters $\hat{\beta}_{i}$ 's are the unfolding parameters. In this case $k=2$ in the contact equivalence (1.2). We allow orientation preserving mixing of $\lambda$ and $\mu$.

Theorem 5 (a) The generic bifurcation problem of corank two with two bifurcation parameters has normal form

$$
f(x, y, \Lambda)=\left(x^{2}+\epsilon y^{2}+\delta_{3} \lambda x+\delta_{4} \mu y+\delta_{1} \lambda, 2 \epsilon x y-\epsilon \delta_{3} y-\delta_{4} \mu x+\delta_{2} \mu\right) .
$$


It is of codimension 2 with universal unfolding

$$
F(x, y, \Lambda, \hat{\beta})=\left(\begin{array}{c}
x^{2}+\epsilon y^{2}+\left(\delta_{3} \lambda+\hat{\beta}_{1}\right) x+\left(\delta_{4} \mu+\hat{\beta}_{2}\right) y+\delta_{1} \lambda \\
2 \epsilon x y-\epsilon\left(\delta_{3} \lambda+\hat{\beta}_{1}\right) y-\left(\delta_{4} \mu+\hat{\beta}_{2}\right) x+\delta_{2} \mu
\end{array}\right) .
$$

(b) The generic gradient bifurcation problem of corank two with two bifurcation parameters has normal form

$$
f(x, y, \Lambda)=\left(x^{2}+\epsilon y^{2}+\delta_{1} \lambda x+\delta \mu 2 \epsilon x y-\epsilon \delta_{1} \lambda y+\delta_{2} \lambda+m \mu\right) .
$$

It is of topological codimension 1 with universal unfolding,

$$
F(x, y, \Lambda, \hat{\beta})=\left(\begin{array}{c}
x^{2}+\epsilon y^{2}+\delta_{1} \lambda x+\delta \mu \\
2 \epsilon x y-\epsilon \delta_{1} \lambda y+\delta_{2} \lambda+m \mu+\hat{\beta}
\end{array}\right)
$$

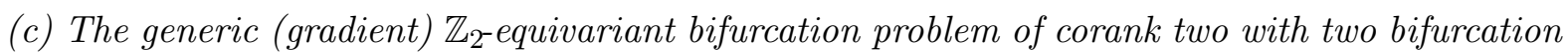
parameters is of codimension 0, equal to its universal unfolding,

$$
f(x, y, \Lambda)=\left(\begin{array}{c}
x^{2}+\epsilon y^{2}+\delta_{2} \mu x+\delta_{1} \lambda \\
2 \epsilon x y-\epsilon \delta_{2} \mu y
\end{array}\right) .
$$

Proof. The proofs follow the pattern of Theorem 3.1. In this case the tangent spaces are submodules of $\overrightarrow{\mathcal{O}}_{(\lambda, \mu)}$ over $\mathcal{O}_{(\lambda, \mu)}$ and we need consider the generators $\bar{\alpha}_{\lambda}$ and $\bar{\alpha}_{\mu}$ in (1.3).

(a) We use the terms of order one of the paths to show that the problem has lowest codimension outside of a codimension one variety (in terms of the coefficient of the Taylor series expansion of the paths): $\bar{\alpha}_{1}(\Lambda)=a \lambda+b \mu+\mathcal{M}_{\lambda}^{2}, \bar{\alpha}_{2}(\Lambda)=c \lambda+d \mu+\mathcal{M}_{\lambda}^{2}, \bar{\beta}(\Lambda)=e \lambda+f \mu+\mathcal{M}_{\lambda}^{2}, \bar{\gamma}(\Lambda)=g \lambda+h \mu+\mathcal{M}_{\lambda}^{2}$.

(b) Using the same Taylor series expansion as in (a) for $\bar{\alpha}_{1}, \bar{\alpha}_{2}$ and $\bar{\beta}$. We need $a$ or $b \neq 0$ and choose $\lambda$ so that $a \neq 0$. The lowest codimension is achieved when

$$
(a d-b c)(d e-c f)\left[\left(c^{2}-\epsilon e^{2}\right) b^{2}-2(c d-\epsilon e f) a b+\left(d^{2}-\epsilon f^{2}\right) a^{2}\right] \neq 0 .
$$

In that case $\delta=\operatorname{sign} a(a d-b c), \delta_{1}=\operatorname{sign} a, \delta_{2}=\operatorname{sign} a(a d-b c)(d e-c f)$ and the modal parameter is equal to $m=a(a f-b e)(a d-b c)^{-2}$.

(c) Using the same Taylor series expansion as in (a) for $\bar{\alpha}_{1}$ and $\bar{\alpha}_{2}$, the normal form (3.16) needs the condition $(a d-b c) \neq 0$.

Our example of Section 4 is a gradient bifurcation, so 3 bifurcation parameters can get us codimension 0 bifurcation problems. In the original work [23] the problem had two parameters. But, even if it had $\mathbb{Z}_{2}$-symmetry, the fact that it had a trivial branch (zero solutions for all values of the parameters) meant that it had infinite codimension (cf. [11] for further discussions on the issue and alternative solutions). By adding a dead load distribution and a non linear elastic foundation controlled by additional parameters we can recover generic diagrams. We denote the bifurcation parameters by $\Lambda=(\lambda, \mu, \nu)$. As previously, the coefficients $\delta_{i}$ are \pm 1 .

Theorem 6 (a) The generic gradient bifurcation problem of corank two with three bifurcation parameters is of codimension 0 with universal unfolding

$$
f(x, y, \Lambda)=\left(\begin{array}{c}
x^{2}+\epsilon y^{2}+\delta_{2} \mu x+\delta_{1} \lambda \\
2 \epsilon x y-\epsilon \delta_{2} \mu y+\delta_{3} \nu
\end{array}\right) .
$$


(b) The generic gradient bifurcation problem of corank two with two bifurcation parameters $(\lambda, \mu)$ with the hierarchy $\lambda>>\mu$, that is, taking $L(\Lambda)=\left(L_{1}(\Lambda), L_{2}(\mu)\right)$ in (1.3), is of topological codimension 1 with universal unfolding

$$
F(x, y, \Lambda, \hat{\beta})=\left(\begin{array}{c}
x^{2}+\epsilon y^{2}+\delta_{2} \lambda x+m \lambda+\delta_{1} \mu \\
2 \epsilon x y-\epsilon \delta_{2} \mu y+\delta_{3} \lambda+n \nu+\hat{\beta}
\end{array}\right)
$$

where $m$ and $n$ are modal parameters satisfying $m n \neq \delta_{3}, m^{2}, n^{2} \neq \epsilon$.

Note that this change of coordinates means that the $\mu$-ordering of the $\lambda$-slices is preserved.

Proof. The proofs follow the pattern of Theorem 3.1.

(a) In this case the tangent spaces are sub-modules of $\overrightarrow{\mathcal{O}}_{(\lambda, \mu, \nu)}$ over $\mathcal{O}_{(\lambda, \mu, \nu)}$ and we consider the generators $\bar{\alpha}_{\lambda}, \bar{\alpha}_{\mu}$ and $\bar{\alpha}_{\nu}$. If the paths is

$$
\left(a_{11} \lambda+a_{12} \mu+a_{13} \nu, a_{21} \lambda+a_{22} \mu+a_{23} \nu, a_{31} \lambda+a_{32} \mu+a_{33} \nu\right)+\overrightarrow{\mathcal{M}}_{\lambda}^{2}
$$

If $\operatorname{det}(a) \neq 0$ we get the generic diagram $(3.17)$.

(b) In this case the tangent spaces have a $\left(\mathcal{O}_{\Lambda}, \mathcal{O}_{\lambda}\right)$-module structure in $\overrightarrow{\mathcal{O}}_{(\lambda, \mu)}$. We also consider the generators $\bar{\alpha}_{\lambda}$ over $\mathcal{O}_{\Lambda}$ and $\bar{\alpha}_{\mu}$ over $\mathcal{O}_{\mu}$, the Derlog part remaining unchanged.

Consider the path $(a \lambda+b \mu, c \lambda+d \mu, e \lambda+f \mu)+\overrightarrow{\mathcal{M}}_{\lambda}^{2}$. It is of the lowest codimension, 1 , if $a$ or $b \neq 0$. We choose $\lambda$ so that $a \neq 0$. Then the conditions for the paths are $\delta_{1}=\operatorname{sign} a(a d-b c)$, $\delta_{2}=\sin a, \delta_{3}=\operatorname{sign} e, m=\frac{c}{|e|}, n=\frac{\delta_{1}(a f-b e)}{(a d-b c)}$.

\section{Examples of Bifurcation Diagrams}

In this section we discuss some concrete examples of bifurcation diagram coming from a simple elastic model with a distribution of loads controlled by a few parameters.

\subsection{Cylindrical Panel}

A cylindrical panel is subject to an axial compression and a load distribution. The configuration domain is $\Omega=[\mu, 0] \times[0,1]$ where $\mu$ represents the aspect ratio of the rectangle. We represent the co-ordinates in $\Omega$ by $(s, t)$. The functions $u$ and $f$ represent the non dimensional values of the vertical displacement and the Airy tension. From the von Kármán-Donnell's shell theory, $u$ and $f$ satisfy

$$
\begin{aligned}
\Delta^{2} u & =-\lambda u_{s s}+[u, f]+d f_{s s}+\nu h, \\
\Delta^{2} f & =-\frac{1}{2}[u, u]-d u_{s s}
\end{aligned}
$$

where we denote partial derivatives using the convention $u_{s}=\frac{\partial u}{\partial s}$. The operator $\Delta$ represents the usual Laplacian in the plane and $[u, v]=u_{s s} v_{t t}+u_{t t} v_{s s}-2 u_{s t} v_{s t}$ is the Monge-Ampère's operator. The parameter $d$ is proportional to the inverse of the radius of curvature of the panel, $\lambda$ to the external force exercised on the $t$-side and $h$ represents a load distribution on the panel with $\nu$ proportional to its strength. There are many boundary conditions for $u$ and $f$ in the literature. In [23] the simply supported panel is used: $u=\Delta u=f=\Delta f=0$. The choice of the best boundary 
conditions for a particular experiment is a subject of many discussion. Practically, the question is to decide which mixture of boundary condition (of Neumann, Dirichlet or mixed type) to put on $u$ and $f$. For this work we can choose any boundary conditions that gives the $\mathbb{Z}_{2}$-action on the kernel we described earlier.

Many of the following results can be found in [23]. The first bifurcation values of the parameter from the unstressed plate are $\lambda_{c}=\frac{9}{2} \pi^{2}+d^{2}\left(\frac{9}{2} \pi^{2}\right)^{-1}, \mu_{c}=\sqrt{2}$ and $\nu_{c}=0$. The kernel of the linearisation is two dimensional, generated by $u_{1,1}(s, t)=\sin \frac{\pi s}{\sqrt{2}} \sin \pi t$ and $u_{2,1}(s, t)=\sin \sqrt{2} \pi s \sin \pi t$. We can solve uniquely (4.20) for $f$ as a function of any given $u$ with our boundary conditions: $f(u)=-\Delta^{-2}\left(\frac{1}{2}[u, u]+d u_{s s}\right)$. Then $D f(0) v=-d \Delta^{-2}\left(v_{s s}\right)$ and $D^{2} f(0) v w=-\Delta^{-2}([v, w])$. Replacing in (4.19) we find a fourth order PDE in $u$ :

$$
F(u, \lambda, \mu, \nu)=\Delta^{2} u+\lambda u_{s s}-[u, f(u)]-d(f(u))_{s s}-\nu h=0 .
$$

Note that, when $\nu=0$, the equations are invariant with respect to the reversor $S:(s, t) \mapsto$ $(\mu-s, 1-t)$ and that $u_{1,1}$ is $S$-invariant and $u_{2,1}$ is $S$-equivariant.

The problem is variational because

$$
\Phi(u, \lambda, \mu, \nu)=\frac{1}{2} \int_{0}^{\mu} \int_{0}^{1}\left\{(\Delta u)^{2}+\left[\Delta^{-1}\left(\frac{1}{2}[u, u]+d u_{s s}\right)\right]^{2}-\lambda\left(u_{s}\right)^{2}-2 \nu h u\right\} d s d t
$$

represents the potential function of the plate. With some symmetry on $h$ we can preserve the $\mathbb{Z}_{2}$-symmetry of the bifurcation equations.

As an extension of the result in [23], following a classical Lyapounov-Schmidt process ([14]), we find the following.

Proposition 7 (a) The 2-jet of the bifurcation function for (4.21) is

$$
\left(\begin{array}{c}
A x^{2}+C y^{2}+\left(a_{1} \lambda+a_{2} \mu+a_{3} \nu\right) x+a_{4} \nu y+c_{1} \nu+\text { h.o.t. } \\
2 C x y+\left(b_{1} \lambda+b_{2} \mu+b_{3} \nu\right) y+a_{4} \nu x+c_{2} \nu+\text { h.o.t }
\end{array}\right)
$$

where $x u_{1,1}+y u_{2,1}$ is the component of $u$ in the kernel of the linearisation, $(\lambda, \mu, \nu)$ the difference to the bifurcation point $\left(\lambda_{c}, \mu_{c}, 0\right), A=-\frac{162^{\frac{3}{4}}}{9} d, C=-\frac{3642^{\frac{3}{4}}}{45} d, a_{1}=-\frac{\pi^{2}}{2}, a_{2}=4 a_{1}, a_{3}=$, $b_{1}=\frac{3 \sqrt{2}}{4} \pi^{4}-\frac{\sqrt{2}}{27} d^{2}, b_{2}=-3 \sqrt{2} \pi^{4}+\frac{4 \sqrt{2}}{27} d^{2}, b_{3}=, c_{1}=P_{1} h, c_{2}=P_{2} h$ the projections of $h$ on $u_{1,1}$, $u_{2,1}$, respectively.

(b) When $h$ is also $\mathbb{Z}_{2}$-equivariant, the 2-jet of the bifurcation equation (4.22) simplifies to

$$
\left(\begin{array}{c}
A x^{2}+C y^{2}+\left(a_{1} \lambda+a_{2} \mu+a_{3} \nu\right) x+c_{1} \nu+\text { h.o.t. } \\
2 C x y+\left(b_{1} \lambda+b_{2} \mu+b_{3} \nu\right) y+h . o . t
\end{array}\right)
$$

Proof. A general element in $\operatorname{ker} \mathcal{L}$ is given by $\Phi(z)=x u_{1,1}+y u_{2,1}$. The projector $P$ onto ker $\mathcal{L}$ is given by

$$
P u=\left(P_{1} u, P_{2} u\right)=\left(<u_{1,1}, u>,<u_{2,1}, u>\right)
$$

and $Q=I-P$. The bifurcation equation is

$$
g(z, \lambda, \mu, \nu)=P F(\Phi(z)+w(z, \lambda, \mu, \nu), \lambda, \mu, \nu)
$$


where $w$ is the unique solution of

$$
Q F(\Phi(z)+w(z, \lambda, \mu, \nu), \lambda, \mu, \nu)=0 .
$$

Most of the calculation have been done in [23]. The derivatives of $g$ at the origin are given by

$$
\begin{aligned}
g_{z z} & =P F_{u u} \Phi^{2}, \\
g_{z \lambda} & =P F_{u u} \Phi w_{\lambda}+P F_{u \lambda} \Phi=P F_{u \lambda} \Phi=P(\Phi)_{s s}, \\
g_{\nu} & =P F_{\nu}=P h, \\
g_{z \nu} & =P F_{u u} \Phi w_{\nu}+P F_{u \nu} \Phi=P F_{u u} \Phi w_{\nu},\left(F_{u \nu}=0\right) .
\end{aligned}
$$

The results of $(4.24,4.25)$ are in [23] as is the result for $g_{z \mu}$.

Moreover $w_{\nu}$ is given by $\mathcal{L} w_{\nu}+Q h=0$ with the Dirichlet boundary conditions where

$$
\mathcal{L} v=\Delta^{2} v+\lambda v_{s s}-d(D f(0) v)_{s s}=\Delta^{2} v+\lambda v_{s s}+d^{2}\left(\Delta^{-2} v_{s s}\right)_{s s} .
$$

The eigenfunctions of $\mathcal{L}$ are $u_{m, n}(s, t)=\phi_{m}(s) \psi_{n}(t)$ where

$$
\phi_{m}(s)=2^{\frac{1}{4}} \sin \left(\frac{m \pi s}{\sqrt{2}}\right), \quad \psi_{n}(t)=\sqrt{2} \sin (n \pi t) .
$$

The corresponding eigenvalue is

$$
\lambda_{m, n}=\frac{\pi^{2}}{2 m^{2}}\left(m^{2}+2 n^{2}\right)^{2}+d^{2} \frac{2 m^{2}}{\pi^{2}}\left(m^{2}+2 n^{2}\right)^{-1} .
$$

To find $w_{\nu}$ we use Fourier series: $w_{\nu}=\sum_{m, n} a_{m, n} u_{m, n}$. And so

$$
w_{\nu}=-\sum_{m, n} \frac{<Q h, u_{k, m}>}{\lambda_{m, n}} u_{m, n}
$$

with $(m, n) \neq(1,1),(2,1)$.

Note that $\Delta u_{m, n}=-\pi^{2}\left(\frac{1}{2} m^{2}+n^{2}\right) u_{m, n}$, and so $\Delta^{-2} u_{m, n}=\frac{4}{\pi^{4}}\left(m^{2}+2 n^{2}\right)^{-2} u_{m, n}$. Moreover,

$$
F_{u u} v w=-[v, D f(0) w]-[w, D f(0) v]-d\left(D^{2} f(0) v w\right)_{s s} .
$$

We can now calculate (4.27). Its coordinates are

$$
x P_{i} F_{u u} u_{1,1} w_{\nu}+y P_{i} F_{u u} u_{2,1} w_{\nu},
$$

$i=1,2$. Now, for $l=1,2$,

$$
F_{u u} u_{l, 1} w_{\nu}=-\sum_{m, n} a_{m, n}\left\{\left[u_{l, 1}, f^{\prime}(0) u_{m, n}\right]+\left[u_{m, n}, f^{\prime}(0) u_{l, 1}\right]+d\left(f^{\prime \prime}(0) u_{l, 1} u_{m, n}\right)_{s s}\right\} .
$$

Because

$$
\left[u_{l, 1}, f^{\prime}(0) u_{m, n}\right]=-d\left[u_{l, 1}, \Delta^{-2}\left(u_{m, n}\right)_{s s}\right]=\frac{d m^{2}}{2 \pi^{2}}\left(\frac{m^{2}}{2}+n^{2}\right)^{-2}\left[u_{l, 1}, u_{m, n}\right]
$$


$\left[u_{m, n}, f^{\prime}(0) u_{l, 1}\right]=\frac{d l^{2}}{2 \pi^{2}}\left(\frac{l^{2}}{2}+1\right)^{-2}\left[u_{m, n}, u_{l, 1}\right]$ and $f^{\prime \prime}(0) u_{l, 1} u_{m, n}=-\Delta^{2}\left[u_{l, 1}, u_{m, n}\right]$, we get

$$
\begin{aligned}
F_{u u} u_{l, 1} w_{\nu}= & -\frac{d}{2 \pi^{2}} \sum_{m, n} a_{m, n}\left\{m^{2}\left(\frac{1}{2} m^{2}+n^{2}\right)^{-2}+l^{2}\left(\frac{1}{2} l^{2}+1\right)\right\}\left[u_{m, n}, u_{l, 1}\right] \\
& +d \sum_{m, n} a_{m, n}\left(\Delta^{-2}\left[u_{l, 1}, u_{m, n}\right]\right)_{s s} .
\end{aligned}
$$

Note that

$$
2\left[u_{m, n}, u_{k, l}\right]=\pi^{4}\left(m^{2} l^{2}+n^{2} k^{2}\right) \phi_{m} \phi_{k} \psi_{n} \psi_{l}-4 \phi_{m}^{\prime} \phi_{k}^{\prime} \psi_{n}^{\prime} \psi_{l}^{\prime}
$$

and $\int_{0}^{1} \sin m t \cos n t d t=\frac{m}{\pi^{2}\left(m^{2}-n^{2}\right)}, m-n$ is even (non zero), 0 otherwise.

\subsection{Paths and Normal Forms}

In this section we briefly discuss the reduction of $(4.22,4.23)$ to the normal form in the path formulation for gradient problems with and without $\mathbb{Z}_{2}$-equivariance. Note that the $\mathbb{Z}_{2}$-symmetry of the nonlinear terms when $\nu=0$ means that the cubic terms only need rescaling. Moreover, because we are only interested in generic paths, it is enough to concern ourselves with the linear terms in the paths.

Proposition 8 Without symmetry, the potential

$$
\frac{1}{3} A x^{3}+C x y^{2}+\frac{1}{2} a_{1}(\Lambda) x^{2}+a_{2}(\Lambda) x y+\frac{1}{2} b_{3}(\Lambda) y^{2}+c_{1}(\Lambda) x+c_{2}(\Lambda) y
$$

can be cast into

$$
\frac{1}{3} x^{3}+\epsilon x y^{2}+\frac{1}{2} \alpha_{1}(\Lambda)\left(x^{2}-\epsilon y^{2}\right)+\alpha_{2}(\Lambda) x+\beta_{1}(\Lambda) y
$$

where $\epsilon=\operatorname{sign}(C), \alpha_{1}=\frac{1}{2} \frac{A^{-2 / 3}}{C}\left(a_{1}-A b_{3}\right), \alpha_{2}=A^{-1 / 3} c_{1}$ and $\beta_{1}=A^{1 / 6}|C|^{-1 / 2} c_{1}$ (modulo second order terms) via a change of coordinates preserving the gradient structure of the bifurcation equation. Recall that we have assumed that $A>0$.

In case the potential is $\mathbb{Z}_{2}$-symmetric,

$$
\frac{1}{3} A x^{3}+C x y^{2}+\frac{1}{2} a_{1}(\Lambda) x^{2}+\frac{1}{2} b_{3}(\Lambda) y^{2}+c_{1}(\Lambda) x
$$

can be cast into

$$
\frac{1}{3} x^{3}+\epsilon x y^{2}+\frac{1}{2} \alpha_{1}(\Lambda)\left(x^{2}-\epsilon y^{2}\right)+\alpha_{2}(\Lambda) x
$$

where $\epsilon=\operatorname{sign}(C), \alpha_{1}=\frac{1}{2} \frac{A^{-2 / 3}}{C}\left(a_{1}-A b_{3}\right)$ and $\alpha_{2}=A^{-1 / 3} c_{1}$ modulo second order terms.

Proof. Without symmetry we use the change of coordinate in $(x, y)$ given by $x \mapsto \gamma(x+\alpha)$ and $y \mapsto \delta(y+\beta)$ where $\gamma, \delta, \alpha, \beta$ are to be determined. Some algebra shows that $A \gamma^{3}=1$, $C \gamma \delta^{2}=\epsilon, \alpha_{1}=a_{1} \gamma^{2}+2 \alpha=-\epsilon b_{3} \delta^{2}-2 \alpha, 0=a_{2} \gamma \delta+2 \epsilon \beta, \alpha_{2}=a_{1} \gamma^{2} \alpha+a_{2} \gamma \delta \beta+c_{1} \gamma$ (modulo quadratic terms) and $\beta_{1}=a_{2} \gamma \delta \alpha+b_{3} \delta^{2} \beta+c_{2} \delta$ (also modulo quadratic terms). Therefore, choosing $\alpha=-\frac{1}{4}\left(a_{1} \gamma^{2}+\epsilon b_{3} \delta^{2}\right)$ and $\beta=-\frac{\epsilon}{2} a_{2} \gamma \delta$, we get $\alpha_{1}(\Lambda)=\frac{1}{2} \frac{A^{-2 / 3}}{C}\left(a_{1}-A a_{2}\right), \alpha_{2}(\Lambda)=A^{-1 / 3} c_{1}$ and $\beta_{1}=A^{1 / 6}|C|^{-1 / 2} c_{1}$ modulo second order terms.

With the $\mathbb{Z}_{2}$-symmetry we use the changes $x \mapsto \gamma(x+\alpha)$ and $y \mapsto \delta y$ that preserve the symmetry. We conclude as previously. 


\subsection{Diagrams for Gradient Problems}

We are considering two cases: a one parameter gradient problem (the parameter must be the load $\nu$ for finite codimension) and a two parameter $\mathbb{Z}_{2}$-equivariant problem with $\nu$ and $\lambda$ (both parameter deal with varying loads).

\subsubsection{One parameter generic gradient diagram}

There are three types of one parameter unperturbed diagrams (3.12). When $\epsilon=-1$ there are two unstable branches (for all $m$ ). When $\epsilon=1$, there are no branches if $|m|>1$ or four branches if $|m|<1$, only one of them stable. Thereafter we describe the transition varieties. They represent the values of the unfolding parameters where bifurcation diagrams are not equivalent.

Let $F(z, \lambda, \hat{\beta})=\nabla G_{0}(z, A(\lambda, \hat{\beta}))$ be a miniversal unfolding of a one parameter gradient bifurcation problem (3.12). Recall that there are three types of transition varieties with one bifurcation parameter problems $([15])$ :

1. $\mathcal{B}$ (when the perturbed diagram has a bifurcation point), of equation

$$
F(z, \lambda, \hat{\beta})=0, \quad \operatorname{rk}\left(F_{z}(z, \lambda, \hat{\beta}), F_{\lambda}(z, \lambda, \hat{\beta})\right) \leq 1 .
$$

2. $\mathcal{H}$ (when the perturbed diagram has an hysteresis point), of equations

$$
F(z, \lambda, \hat{\beta})=0, \quad\left|F_{z}(z, \lambda, \hat{\beta})\right|=0
$$

where $\left|F_{z}\right|$ indicates the determinant of the Jacobian matrix $F_{z}$, and

$$
F_{z z}(z, \lambda, \hat{\beta})(v, v) \in \operatorname{ran}\left(F_{z}(z, \lambda, \hat{\beta})\right), \quad v \in \operatorname{ker}\left(F_{z}(z, \lambda, \hat{\beta})\right) .
$$

3. $\mathcal{D} \mathcal{L}$ (when the perturbed diagram has two fold points at the same value of $\lambda$ ), of equations

$$
F(z, \lambda, \hat{\beta})=F(\bar{z}, \lambda, \hat{\beta})=0, \quad\left|F_{z}(z, \lambda, \hat{\beta})\right|=\left|F_{z}(\bar{z}, \lambda, \hat{\beta})\right|=0,
$$

for $z \neq \bar{z}$.

After some calculations, they are given by the following Taylor series expansions:

$$
\begin{aligned}
\mathcal{B}: & \hat{\beta}_{2}=\delta m \hat{\beta}_{1}+\delta m \frac{\left(m^{4}-8 \epsilon m^{2}-3\right)}{\left(m^{2}-\epsilon\right)^{2}} \hat{\beta}_{1}^{2}+\ldots \\
\mathcal{H}: & \hat{\beta}_{2}=0, \quad m=0 \\
& \hat{\beta}_{2}^{+}=\delta m \hat{\beta}_{1}-\frac{1}{4} \delta m \hat{\beta}_{1}^{2}+\ldots, \quad m \neq 0 \\
& \hat{\beta}_{2}^{-}=\delta m \hat{\beta}_{1}+\frac{3}{4} \delta m \hat{\beta}_{1}^{2}+\ldots, \quad m \neq 0 \\
& \hat{\beta}_{2}^{ \pm}=\delta m \hat{\beta}_{1}-\frac{3}{8}(\delta m \pm \sqrt{3}) \hat{\beta}_{1}^{2}+\ldots, \quad \epsilon \neq-1 \\
\mathcal{D} \mathcal{L} \quad: \quad & \hat{\beta}_{2}=\delta m \hat{\beta}_{1}+(B-\delta m A) \hat{\beta}_{1}^{2}+\ldots
\end{aligned}
$$

where the constants $A$ and $B$ are given by $A=0.028374, \delta_{1} B= \pm 0.473955, \pm 0.06582$ when $\epsilon-1$ (4 branches), or $A=-1.247408, \delta_{1} B= \pm 1.4457375, \pm 1.4773705$ and $A=-4.56305, \delta_{1} B=$ $\pm 4.276497, \pm 7.681799$ when $\epsilon-1$ ( 8 branches). 


\subsubsection{Two parameters generic gradient diagram}

When the external load $h$ satisfy the $\mathbb{Z}_{2}$-symmetry, the bifurcation equations simplify. In the following figures we represent the solution set of $F_{0}^{\mathbb{Z}_{2}}$ in (2.6) as its projection on the $\left(\alpha_{1}, \alpha_{2}\right)$ plane. The pattern of solutions is the same in each connected component of $\mathbb{R}^{2}-\Delta^{\mathbb{Z}_{2}}$ where $\Delta^{\mathbb{Z}_{2}}$ is the discriminant of $F_{0}^{\mathbb{Z}_{2}}$. Recall that it has two components: $\mathcal{B}_{x}, \mathcal{P}_{\kappa}$.

The squares represent $\mathbb{Z}_{2}$-symmetric solutions $(y=0)$, circles the solutions without any symmetry (appearing as $\mathbb{Z}_{2}$-symmetric pairs). A black square $\mathbf{\square}$ or circle $\bullet$ represent solutions with eigenpair of negative real parts $(-,-)$, crossed squares $\otimes$ or circles $\otimes$ with eigenpair of positive real parts $(+,+)$ as white squares $\square$ or circles $\circ$ are saddle points. Bifurcation diagrams can be represented by paths through the figure.
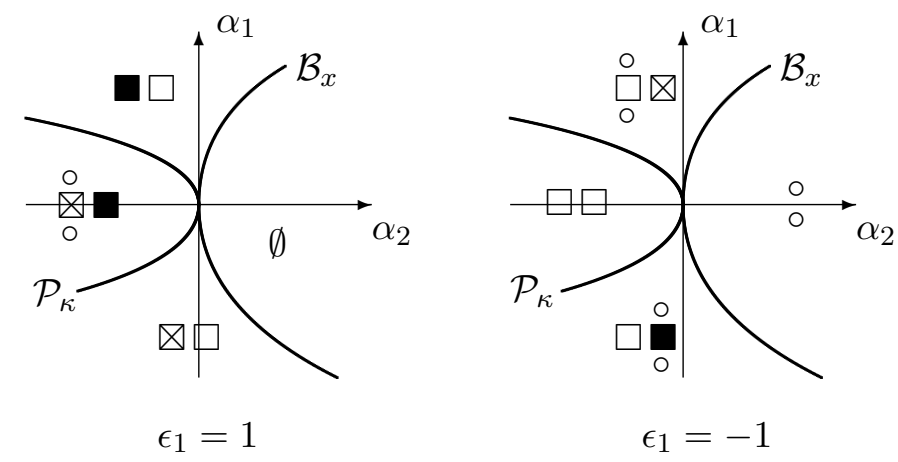

Figure 1: Solutions of $F_{0}^{\mathbb{Z}_{2}}(z, \alpha)=0$ as a function of $\alpha$

\section{References}

[1] V.I. Arnold. Wavefront evolution and equivariant Morse lemma. Comm. Pure. App. Math. 29 (1976), 557-582.

[2] T.J. Bridges, J.E. Furter. Singularity theory and equivariant symplectic maps. Springer Lectures Notes 1558, Heidelberg 1993.

[3] J.W. Bruce, D.L. Fidal. Generic Functions on Bifurcation varieties I. Preprint, unpublished.

[4] J.W. Bruce, A. du Plessis, L.C. Wilson. Discriminants and Liftable Vector Fields. Jour. Alg. Geom. 3 (1994), 725-753.

[5] C.J. Cheng, X.C. Shang. Mode Jumping of Simply Supported Rectangular Plates on Non-Linear Elastic Foundations. Int. J. Non-Linear Mechanics 32 (1997), 161-172.

[6] J. Damon. Deformation of Sections of Singularities and Gorenstein Surface Singularities. Am. Jour. of Maths. 109 (1987), 695-722.

[7] G. Dangelmayer, D. Armbruster. Classification of $\mathbb{Z}_{2}$-equivariant imperfect bifurcations with corank 2. Proc. Lond. Math. Soc. 46 (1983), 517-546.

[8] J.E. Furter, A.M. Sitta, I. Stewart. Algebraic path formulation for equivariant bifurcation problems. Math. Proc. Camb. Phil. Soc. 124 (1998), 275-304. 
[9] J.E. Furter. Geometric Path Formulation for Bifurcation Problems. Journal of Natural Geometry 12, London 1997.

[10] J.E. Furter, A.M. Sitta. Path Formulation for Bifurcation Problems. Preprint.

[11] J.E. Furter, A.M. Sitta. Multiparameter Bifurcation Problem and Finite Determinacy: a $\mathbb{Z}_{2}-$ Equivariant Corank 2 Example. Submitted for publication.

[12] J.E. Furter, A.M. Sitta. A Note on the Non-degenerate Umbilics and the Path Formulation for Bifurcation Problems. Proc. Roy. Soc. Edin. 134A (2004) 1115-1126.

[13] M. Golubitsky, D.G. Schaeffer. A theory for imperfect bifurcation via singularity theory. Comm. Pure. Appl. Math. 32 (1979), 21-98.

[14] M. Golubitsky, D.G. Schaeffer. Singularities and groups in bifurcation theory I. Applied Math. Sciences 51, Springer Verlag, New York 1985.

[15] M. Golubitsky, I. Stewart and D.G. Schaeffer. Singularities and groups in bifurcation theory II. Applied Math. Sciences 69, Springer Verlag, New York 1988.

[16] W.J.F. Govaerts. Numerical Methods for Bifurcations of Dynamical Equilibria. SIAM, Philadelphia, 2000.

[17] A. Lari-Lavassami. Multiparameter bifurcation with symmetry via singularity theory. PhD. Thesis, Ohio State University, 1990.

[18] E.J.N. Looijenga. Isolated Singular Points of Complete Intersections. LMS Lecture Notes 17, Cambridge University Press, 1984.

[19] D. Mond, J. Montaldi. Deformations of Maps on Complete Intersections, Damon's $\mathcal{K}_{V^{-}}$ equivalence and Bifurcations, in Singularities, Lille 1991 (J.P. Brasselet ed.). LMS Lecture Notes 201, Cambridge University Press, Cambridge 1994, 263-284.

[20] J. Montaldi. The Path Formulation of Bifurcation Theory, in Dynamics, Bifurcation and Symmetry: New Trends and New Tools. (P. Chossat ed.). Nato ASI Series 437, Kluwer, Dordrecht 1994, 259-278.

[21] M. Peters. Multiparameter bifurcation problems. PhD Thesis, University of Warwick, 1991.

[22] K. Saito. Theory of logarithmic differential forms and logarithmic vectorfields. J. Fac. Sci. Univ. Tokyo Sec 1A 27 (1980), 265-291.

[23] B. Wu. Constructive analysis of buckling mode interactions with single $\mathbb{Z}_{2}$-symmetry. Int. Jour. Non-Linear Mech. 34 (1999), 671-683.

[24] C. Zuppa. Bifurcations imparfaites de type potentiel et D-équivalences de déformations des fonctions. Duke Math. Jour. 51 (1984), 729-763. 